\title{
HEAVY SUBGRAPH CONDITIONS FOR LONGEST CYCLES TO BE HEAVY IN GRAPHS
}

\author{
BinlONG Li ${ }^{a, b, 1}$ AND ShengGui ZhANG ${ }^{a, 2}$ \\ ${ }^{a}$ Department of Applied Mathematics \\ Northwestern Polytechnical University \\ Xi'an, Shaanxi 710072, P.R. China \\ ${ }^{b}$ European Centre of Excellence NTIS \\ 30614 Plzeñ, Czech Republic \\ e-mail: libinlong@mail.nwpu.edu.cn \\ sgzhang@nwpu.edu.cn
}

\begin{abstract}
Let $G$ be a graph on $n$ vertices. A vertex of $G$ with degree at least $n / 2$ is called a heavy vertex, and a cycle of $G$ which contains all the heavy vertices of $G$ is called a heavy cycle. In this note, we characterize graphs which contain no heavy cycles. For a given graph $H$, we say that $G$ is $H$-heavy if every induced subgraph of $G$ isomorphic to $H$ contains two nonadjacent vertices with degree sum at least $n$. We find all the connected graphs $S$ such that a 2 -connected graph $G$ being $S$-heavy implies any longest cycle of $G$ is a heavy cycle.
\end{abstract}

Keywords: heavy cycles, heavy subgraphs.

2010 Mathematics Subject Classification: 05C45, 05C07, 05C38.

\section{REFERENCES}

[1] B. Bollobás and G. Brightwell, Cycles through specified vertices, Combinatorica 13 (1993) 147-155. doi:10.1007/BF01303200

[2] J.A. Bondy and U.S.R. Murty, Graph Theory with Applications (Macmillan, London and Elsevier, New York, 1976). doi:10.1007/978-1-349-03521-2

\footnotetext{
${ }^{1}$ Supported by the project NEXLIZ - CZ.1.07/2.3.00/30.0038.

${ }^{2}$ Supported by the NSFC (11271300). Corresponding author.
} 
[3] G. Fan, New sufficient conditions for cycles in graphs, J. Combin. Theory Ser. B 37 (1984) 221-227. doi:10.1016/0095-8956(84)90054-6

[4] R. Shi, 2-neighborhoods and hamiltonian conditions, J. Graph Theory 16 (1992) 267-271.

doi:10.1002/jgt.3190160310

Received 18 February 2015

Revised 13 July 2015

Accepted 13 July 2015 